\title{
Publisher Correction: Clonal replacement and heterogeneity in breast tumors treated with neoadjuvant HER2-targeted therapy
}

\author{
Jennifer L. Caswell-Jin (1) ', Katherine McNamara (1) 1,2,3, Johannes G. Reiter (1) 4, Ruping Sun (1) 1,2,3, Zheng Hu 1,2,3, \\ Zhicheng Ma1,2,3, Jie Ding ${ }^{1,2,3}$, Carlos J. Suarez ${ }^{5}$, Susanne Tilk ${ }^{6}$, Akshara Raghavendra7, Victoria Forte ${ }^{8,9}$, \\ Suet-Feung Chin (10) ${ }^{10}$, Helen Bardwell ${ }^{10}$, Elena Provenzano ${ }^{11}$, Carlos Caldas (10) ${ }^{10}$, Julie Lang (iD ${ }^{9,12}$, Robert West ${ }^{5}$, \\ Debu Tripathy (iD ${ }^{7}$, Michael F. Press ${ }^{9,13}$ \& Christina Curtis (iD ${ }^{1,2,3}$
}

Correction to: Nature Communications https://doi.org/10.1038/s41467-019-08593-4, published online 08 February 2019.

The original version of this Article omitted from the Author Contributions statement that 'R.S. and J.G.R. contributed equally to this work.' This has been corrected in both the PDF and HTML versions of the Article.

Published online: 30 May 2019

Open Access This article is licensed under a Creative Commons Attribution 4.0 International License, which permits use, sharing, adaptation, distribution and reproduction in any medium or format, as long as you give appropriate credit to the original author(s) and the source, provide a link to the Creative Commons license, and indicate if changes were made. The images or other third party material in this article are included in the article's Creative Commons license, unless indicated otherwise in a credit line to the material. If material is not included in the article's Creative Commons license and your intended use is not permitted by statutory regulation or exceeds the permitted use, you will need to obtain permission directly from the copyright holder. To view a copy of this license, visit http://creativecommons.org/licenses/by/4.0/.
\end{abstract}

(C) The Author(s) 2019

\footnotetext{
${ }^{1}$ Department of Medicine, Division of Oncology, Stanford University School of Medicine, Stanford 94305 California, United States. ${ }^{2}$ Department of Genetics, Stanford University School of Medicine, Stanford 94305 CA, USA. ${ }^{3}$ Stanford Cancer Institute, Stanford University School of Medicine, Stanford 94305 CA, USA. ${ }^{4}$ Canary Center for Cancer Early Detection, Department of Radiology, Stanford University School of Medicine, Palo Alto 94305 CA, USA. ${ }^{5}$ Department of Pathology, Stanford University School of Medicine, Stanford 94305 CA, USA. ${ }^{6}$ Department of Biology, Stanford University, Stanford 94305 CA, USA. 7 Department of Breast Medical Oncology, The University of Texas MD Anderson Cancer Center, Houston 77030 TX, USA. ${ }^{8}$ Maimonides Medical Center, Brooklyn 11219 NY, USA. ${ }^{9}$ Norris Comprehensive Cancer Center, Los Angeles 90033 CA, USA. ${ }^{10}$ Cancer Research UK Cambridge Institute, Department of Oncology, University of Cambridge, Cambridge CB2 ORE, UK. ${ }^{11}$ Cambridge Experimental Cancer Medicine Centre and NIHR Cambridge Biomedical Research Centre, Cambridge University Hospitals NHS Foundation Trust, Cambridge CB2 OQQ, UK. ${ }^{12}$ Department of Surgery, Keck School of Medicine, University of Southern California, Los Angeles 90333 CA, USA. ${ }^{13}$ Department of Pathology, Keck School of Medicine, University of Southern California, Los Angeles 90033 CA, USA. These authors contributed equally: Jennifer L. Caswell-Jin, Katherine McNamara, Johannes G. Reiter, Ruping Sun. Correspondence and requests for materials should be addressed to C.C. (email: cncurtis@stanford.edu)
} 